\title{
The Effect of Novel Low Energy Pulsed Light Combined with Galvanic Energy for Home-Use Hair Removal of Dark Skin
}

\author{
Michael H. Gold1, Hela Goren ${ }^{2}$ \\ ${ }^{1}$ Tennessee Clinical Research Center, Nashville, USA \\ ${ }^{2}$ Home Skinovation Ltd., Yokneam, Israel \\ Email:Rgoren4@gmail.com
}

Received 24 September 2015; accepted 28 November 2015; published 1 December 2015

Copyright (C) 2015 by authors and Scientific Research Publishing Inc.

This work is licensed under the Creative Commons Attribution International License (CC BY). http://creativecommons.org/licenses/by/4.0/

(c) (i) Open Access

\begin{abstract}
Background and Objectives: Permanent reduction of unwanted hair on skin types V and VI is the most challenging procedure among all hair removal technologies based on selective absorption of light or laser. The objective of this study is to evaluate the safety and efficacy of a low energy pulsed-light device combined with galvanic energy, intended for home-use hair removal on dark skin. Materials and Methods: Fifteen women with skin types V and VI and dark terminal hair in axillaarea self-administrated 6 treatments at 2 week intervals, using a hand-held IPL combined with galvanicenergy device, using HPL (Home Pulsed Light) technology. Hair count and photographs were performed pre-treatment and 1 and 3 months after the last sixth treatment. Adverse events and subject satisfaction scores were recorded. Results: All patients showed a positive clinical response to treatment, with reduction of unwanted hair. Hair counts were significantly reduced by $57.3 \% 1$ month following last treatment and by $44.5 \% 3$ months following last treatment. No adverse events were recorded. Subject satisfaction scores of the device usability and the treatment outcome were high. Conclusions: Low energy pulsed light combined with galvanic energy may be applied safely and effectively for at-home hair removal for people with dark skin types V and VI.
\end{abstract}

\section{Keywords}

Hair Removal, Dark Skin, Home-Use, IPL, Galvanic Energy

\section{Introduction}

Long-term removal of unwanted hair is the most popular skin treatment worldwide. Over the past decade, various light and laser sources have been advocated for use in the office setting with long term results [1]-[3]. Selec-

How to cite this paper: Gold, M.H. and Goren, H. (2015) The Effect of Novel Low Energy Pulsed Light Combined with Galvanic Energy for Home-Use Hair Removal of Dark Skin. Journal of Cosmetics, Dermatological Sciences and Applications, 5, 283-290. http://dx.doi.org/10.4236/jcdsa.2015.54034 
tive photothermolysis was established as the mechanism of action of optical sources of light and laser for the selective destruction of hair follicles [4] [5]. However, efficacy and safety of these technologies presented a challenge when dark skin was treated [6]. The disadvantages of in-office based hair removal methods called for the establishment of home-use devices of laser and light sources that enable people to enjoy permanent hair reduction in the comfort of their own home [7]-[11]. The challenge of treating dark skin safely and effectively at home environment still exists, as low fluence light source has to be deployed to protect the dark skin without affecting efficacy of the long-term hair removal. In an attempt to increase absorption of light in the hair melanin, continuous galvanic current has been added. The galvaniccurrent applied that may be as low as $200 \mu \mathrm{A}$ is widely used in cosmetic treatments for a few decades [12]. Such a low direct electrical current is hardly perceptible and may affect temporarily several skin structures, including pore dilation. Dilation of the pores at the skin surface exposes a bigger portion of the hair shaft to the optical energy, thus increasing the absorption and enabling a low fluence to be efficacious. The combination of radiofrequency electrical current and intense pulsed light (IPL) energy has been previously reported for in-office system [13]. The current study is the first attempt to combine the electrical and optical energies in a home-use device in an attempt to treat dark skin safely and effectively.

\section{Materials and Methods}

\subsection{Device and Technology}

The Infinity Device is a small hair removal home-use device, employing the technology of electrical Home Pulsed Light (eHPL ${ }^{\mathrm{TM}}$ ) (Home Skinovation, Yokneam, Israel). It is a portable hand-held device with spot size of $1 \times 3 \mathrm{~cm}$, combining low energy IPL (475 $-1200 \mathrm{~nm} ; 3-5 \mathrm{~J} / \mathrm{cm}^{2}$ ) and $200 \mu \mathrm{A}$ galvanic energy (Figure 1). IPL may be applied in the rate of 1 pulse every 1 - 2 sec in 5 different levels according to individual skin response, following a test spot. The electrical current is a continuous direct current that depends on full contact with the skin. Failure to have this contact results in automatic inactivation of the galvanic energy by a built-in safety mechanism. This mechanism also inactivates IPL if full contact with the skin is not achieved.

\subsection{Subjects}

Seventeen females, 21 - 60 years old with Fitzpatrick skin type VI who had black hair in their axillae and met the inclusion and exclusion criteria were recruited to the study. Subjects were retained by in office recruitment of existing patients who would be a candidate for hair removal through an IRB (Investigational Review Board) approval study. Fifteen subjects have completed the study. All subjects signed an informed consent form.

\subsection{Acceptance Criteria}

Inclusion criteria included healthy females between 21 - 60 years of age with skin type VI, having unwanted

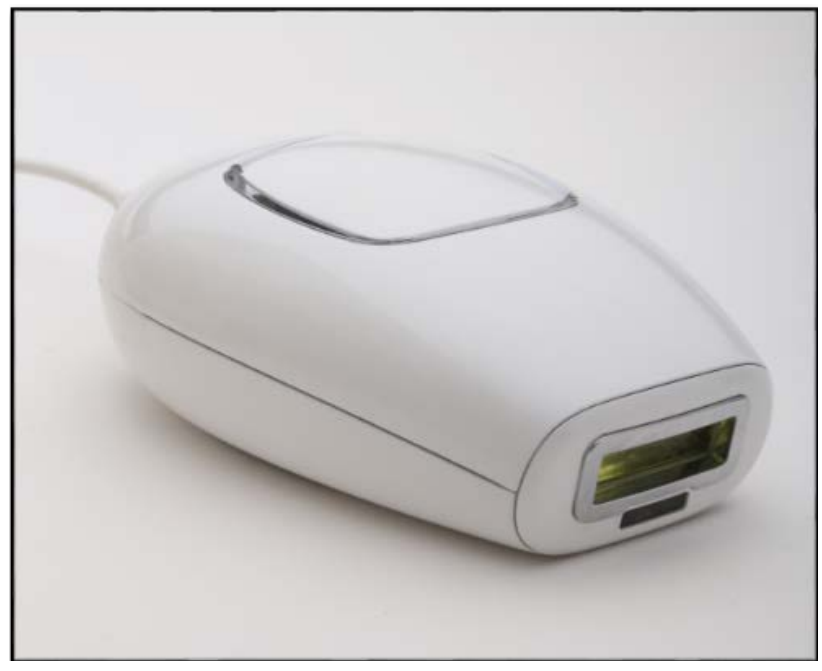

Figure 1. The Infinity Device. 
black hair in their axillae and willingness to follow the study protocol. Exclusion criteria included pregnancy and lactating throughout the study period, concurrent severe diseases and history of malignancy, diseases that may be stimulated by light, such as epilepsy and lupus, scarring, inflammation or infection of the area to be treated, history of skin disorders such as keloids and abnormal wound healing, a pacemaker or internal defibrillator device, poorly controlled endocrine disorders as diabetes or PCO, history of bleeding coagulopathies or constant use of anticoagulants, previous photo-epilation hair removal or waxing and plucking in the treatment area during the last three months, history of known photosensitization or the use of a medication known to induce photosensitization; tanned skin in the treatment area within four weeks of the study, and presence of tattoo in the treatment area.

\subsection{Study Design}

Subjects were given the Infinity device and the instructions for use and were asked to perform the first selftreatment in the investigator's clinic in the supervision of a qualified trainer who determined the fluence used for each individual following a test spot procedure. Baseline measurements included demographic data, laboratory tests to rule out pregnancy, and initial photography and hair counts in both axillae. Subjects conducted 5 more sessions of self-treatment (total 6) at home at 2 weeks intervals. Follow-up sessions were held in the investigator's clinic 1 , and 3 months following the last treatment. At each follow-up session the treated area was photographed and hair count conducted in a similar way to baseline. Photographs were taken with Nikon D100 camera with 60 -mm micro lens. Hair count was done on a predetermined $2 \times 2 \mathrm{~cm}\left(4 \mathrm{~cm}^{2}\right)$ area, using a small sticker. These stickers have a central cut out area that is a reproducible $2 \times 2 \mathrm{~cm}$ in size. The axillae with the stickers were also photographed to ensure count in the same area. Furthermore, a transparency sheet was placed over each axilla of the subject, and anatomic locations (such as nevi, freckles, tags, scars) were marked to facilitate reproducibility of count area in the various visits. The duration of the entire study for each subject was up to 7 months.

\subsection{Treatment Procedure}

Hair was shaved and treatment was performed by applying light pressure on the treatment area with the Infinity device, to achieve complete contact with the skin and pressing the trigger button. The applicator was then moved to the next spot without overlap and with full coverage of the treated area. Post-treatment care included the application of cold Aloe Vera cream and a moisturizer cream and the patients were instructed to protect the treated areas from sun exposure and tanning throughout the whole study. After each treatment subjects were instructed to evaluateany adverse event and attend the clinic for its assessment. At each follow-up session subjects were asked to rate their satisfaction level in a scale of four: 0 -Not satisfied; 1 -Slightly satisfied; 2 -Satisfied; 3-Very satisfied.

\subsection{Statistics}

Sample size was 30 treatment sites of 15 subjects. Hair count p values were performed using paired t-test analysis. Values $<0.001$ were regarded statistically significant.

\section{Results}

All 15 subjects who enrolled and completed the study were females with an average age of 42 years, with skin type VI that were treated on both axillae for unwanted black hair. Energy levels used (out of 5 levels) were 1 - 3 with a mean value of 2.3 .

Results of the clinical study and demographic data are given in Table 1. The Results show that the mean hair count per at baseline was $53.4 \pm 3.9$. At the 1 month follow-up visit, the mean hair count significantly decreased to 22.8 ( $\mathrm{SD}=4.4$ ), by $57.3 \%$ ( $\mathrm{p}<0.001)$. At the 3 month follow-up visit, the mean hair count was decreased to a lesser degree 29.7 ( $S D=4.8$ ) which was still significantly lower than baseline count in $44.5 \%(\mathrm{p}<0.001)$.

The graphic presentation of hair count and percent hair reduction distribution is given in Figure 2.

Representative photographic illustrations of hair reduction in the two follow-up sessions as compared with baseline photographs are presented in Figure 3. Results of hair reduction on three axillae of three different patients with skin type VI are comparing baseline to 3 month follow-up (Figure 4). Both, hair count results and 
Table 1. Hair count and percent hair reduction distribution at baseline and at 2 follow-up sessions, 1 and 3 months post 6 treatments.

\begin{tabular}{|c|c|c|c|c|c|c|c|}
\hline \multirow{2}{*}{$\begin{array}{l}\text { Subject } \\
\text { ID }\end{array}$} & \multirow{2}{*}{ Axilla } & \multirow{2}{*}{ Age } & \multirow{2}{*}{$\begin{array}{c}\text { Energy } \\
\text { Level }\end{array}$} & \multicolumn{3}{|c|}{ Hair Count per $2 \mathrm{~cm}^{2}$ Mean \pm SD } & \multirow{2}{*}{ Response } \\
\hline & & & & Baseline & 1 Month & 3 Months & \\
\hline 1 & Left & \multirow{2}{*}{50} & 2 & 47 & 30 & 37 & Slight erythema \\
\hline 1 & Right & & 2 & 49 & 28 & 34 & Slight erythema \\
\hline 2 & Left & \multirow{2}{*}{28} & 1 & 49 & 19 & 23 & None \\
\hline 2 & Right & & 1 & 49 & 21 & 25 & None \\
\hline 3 & Left & \multirow{2}{*}{44} & 3 & 55 & 17 & 27 & Slight erythema \\
\hline 3 & Right & & 3 & 53 & 21 & 24 & Slight erythema \\
\hline 4 & Left & \multirow{2}{*}{25} & 3 & 51 & 18 & 27 & Perifollicular edema \\
\hline 4 & Right & & 3 & 55 & 28 & 30 & Perifollicular edema \\
\hline 5 & Left & \multirow{2}{*}{59} & 2 & 49 & 26 & 37 & None \\
\hline 5 & Right & & 3 & 47 & 21 & 37 & None \\
\hline 6 & Left & \multirow{2}{*}{45} & 2 & 48 & 25 & 27 & Perifollicular edema \\
\hline 6 & Right & & 2 & 54 & 26 & 29 & Perifollicular edema \\
\hline 7 & Left & \multirow{2}{*}{33} & 2 & 50 & 21 & 33 & None \\
\hline 7 & Right & & 2 & 57 & 19 & 31 & None \\
\hline 8 & Left & \multirow{2}{*}{42} & 1 & 56 & 28 & 28 & None \\
\hline 8 & Right & & 2 & 57 & 30 & 35 & None \\
\hline 9 & Left & \multirow{2}{*}{21} & 2 & 59 & 27 & 34 & Perifollicular erythema \\
\hline 9 & Right & & 2 & 53 & 27 & 30 & Perifollicular edema \\
\hline 10 & Left & \multirow{2}{*}{54} & 3 & 53 & 25 & 31 & Perifollicular erythema \\
\hline 10 & Right & & 3 & 55 & 27 & 30 & Perifollicular erythema \\
\hline 11 & Left & \multirow{2}{*}{55} & 3 & 47 & 17 & 25 & None \\
\hline 11 & Right & & 3 & 50 & 17 & 23 & None \\
\hline 12 & Left & \multirow{2}{*}{60} & 1 & 55 & 19 & 30 & None \\
\hline 12 & Right & & 1 & 58 & 20 & 23 & None \\
\hline 13 & Left & \multirow{2}{*}{28} & 2 & 59 & 29 & 37 & None \\
\hline 13 & Right & & 2 & 59 & 25 & 32 & None \\
\hline 14 & Left & \multirow{2}{*}{32} & 3 & 51 & 17 & 25 & None \\
\hline 14 & Right & & 3 & 50 & 19 & 22 & None \\
\hline 15 & Left & \multirow{2}{*}{49} & 3 & 57 & 25 & 34 & Perifollicular edema \\
\hline 15 & Right & & 3 & 56 & 21 & 35 & Perifollicular edema \\
\hline Mean Hair Count & & 42.1 & 2.3 & 53.4 & 22.8 & 29.7 & \\
\hline SD & & & & 3.93 & 4.37 & 4.78 & \\
\hline $\begin{array}{l}\text { Mean \% Hair } \\
\text { Reduction }\end{array}$ & & & & & $57.3 \%$ & $44.5 \%$ & \\
\hline p-Value & & & & & $2.3502 \mathrm{E}-24$ & $5.64221 \mathrm{E}-20$ & \\
\hline
\end{tabular}




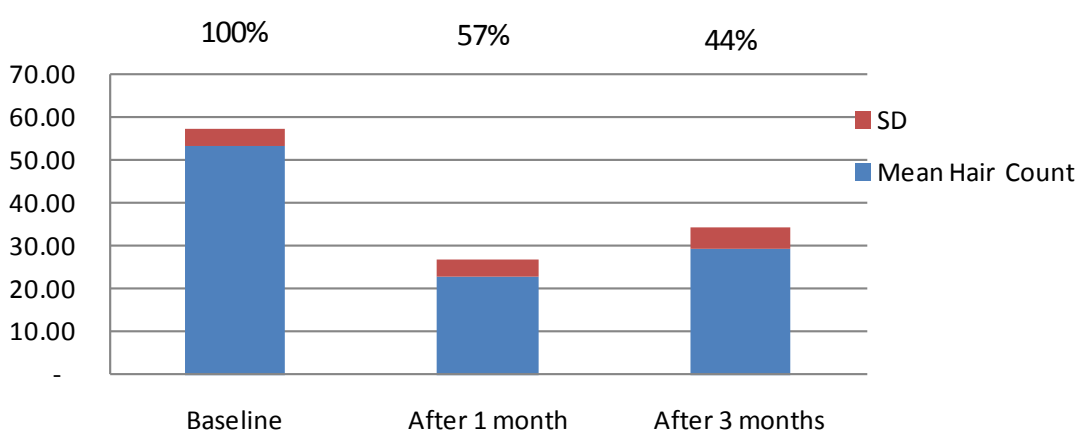

Figure 2. Graphic distribution of hair count and percent hair reduction at baseline and at 2 follow-up sessions, 1 and 3 months post 6 treatments.
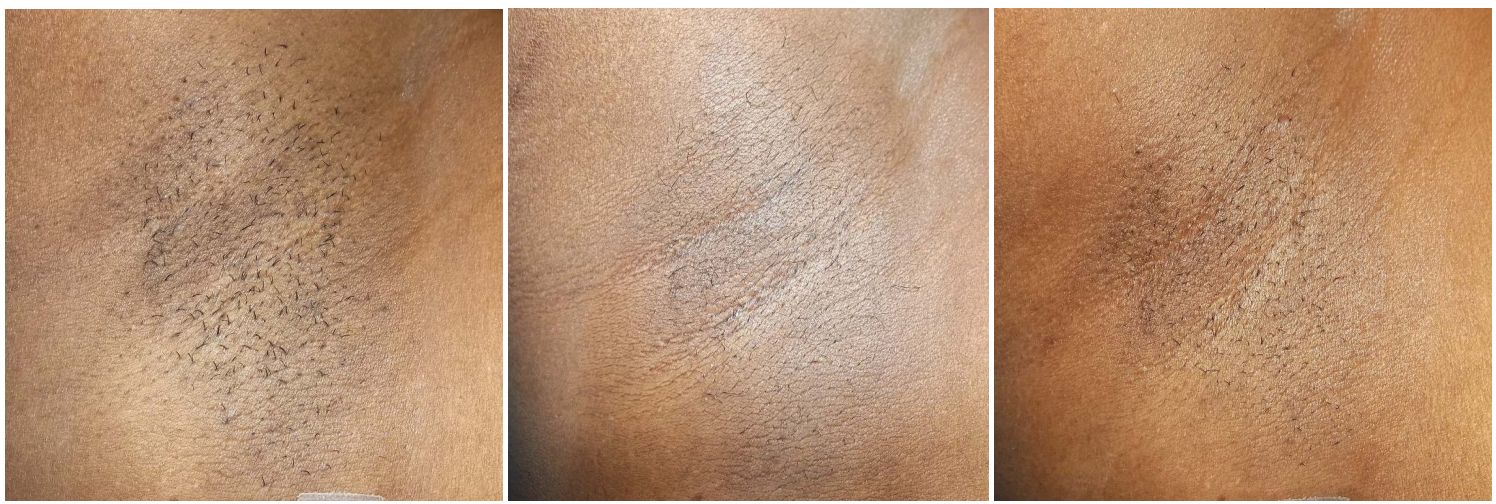

Figure 3. Photographic illustration of hair reduction in axillae of subject 1 at baseline (left), at 1 month follow-up session (center), and 3 months post last treatment (right).

photographs indicate that percent hair reduction after 3 month is smaller than after 1 month.

Subject Satisfaction Score is presented in Table 2. Satisfaction included subjects' impression from the results and from the usability of the device. All subjects rated the usability that reflects ease of use as "very satisfied". Regarding treatment outcome, eight subjects scored their satisfaction as "very satisfied" at the two follow-up sessions. Six subjects rated their satisfaction as "satisfied" after 1 month and 5 of them changed their rating to "very satisfied at the 3 months follow-up sessions. One subject started at "slightly satisfied" at the 1 month follow-up and changed to "satisfied" after 3 months. Altogether, satisfaction rate of both usability of the device and hair reduction outcome is very high (2.5 - 3.0). All subjects expressed their will to purchase the device following the study for home-use treatments and recommend to their friends.

No adverse events were noted during the study. Slightedema in the perifollicular sites was experienced by $33.3 \%$ of the subjects and resolved within 2 hours. This was a desirable and expected response of treatment endpoint.

\section{Discussion}

The current study demonstrated 57.3\% hair reduction 1 month after 6 sessions every other week that was reduced to $44.5 \%$ after 3 months and was also demonstrated in the photographs (Figure 2, Figure 3). These results indicate that a maintenance session may be required after $\sim 2$ months following the treatment regime.

Long-term hair removal performed in-office by optical technologies of lasers and IPL have presented an average efficacy of $60 \%$ - $80 \%$ following a multi-session treatment [3] and with follow-up periods of 1 - 2 years [1] [12]. Home-use devices based on IPL technology proved to be efficacious on body areas [7] [10] and on the face [8] in a similar efficacy as in office-based IPL systems by using lower fluence but more frequent sessions. Thus, $83 \%$ hair reduction was demonstrated on the face after 1 month and $78 \%$ after 3 months following 6 sessions every other week [7]. Percent hair reduction on the body was lower and reached $78 \%$ after 1 month and $72 \%$ after 3 months following 6 sessions [8]. In another study [10] average percent hair reduction observed on body 

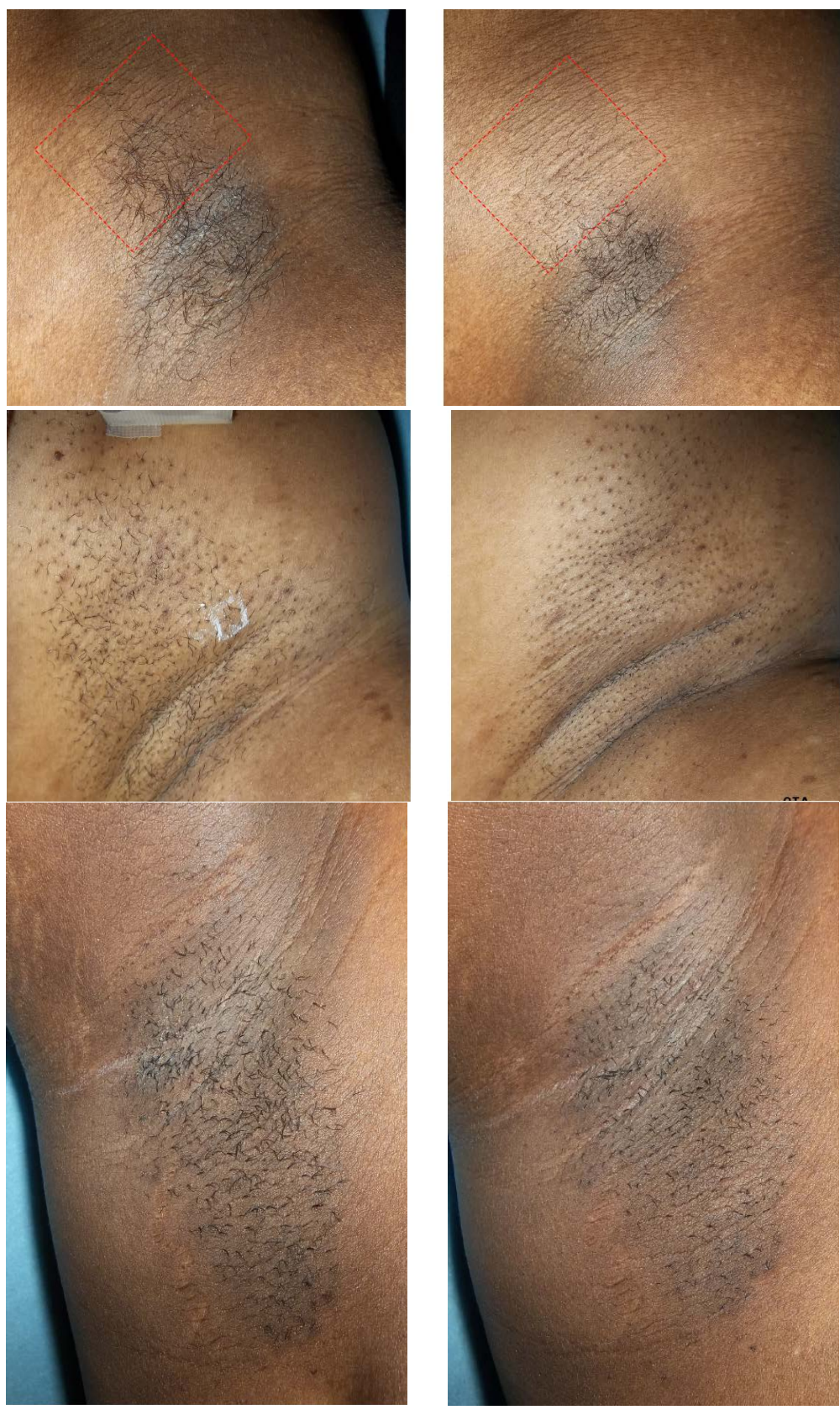

Figure 4. Photographic illustration of hair reduction in axillae of 3 subjects at baseline (Left) and 3 months post last treatment (Right). Top to bottom: subjects $3 R, 10 \mathrm{R}$, and $11 \mathrm{R}$.

areas after 3 months following 3 treatments was 64\%. The three studies were conducted on light skin types, up to Fitzpatrick skin type IV.

In an attempt to adjust the home-use hair removal device to dark skin type (V-VI), lower fluence was applied to the dark skin to ensure safety (energy levels 1 - 3), but efficacy was increased by the simultaneous addition of galvanic energy. Nevertheless, as expected, percent hair reduction was lower than on lighter skin, using a similar device. As the studies described above were conducted with a similar IPL device [7]-[9] on light skin, it is important to note that using lower level energy on dark skin types may require more treatment sessions. Therefore, to achieve similar efficacy to results on lighter skin, it is expected that treatment of darker skin types may require up to 12 treatments every other week. 


\section{Table 2. Subject satisfaction scores.}

\begin{tabular}{|c|c|c|c|c|}
\hline \multirow[t]{2}{*}{$\begin{array}{l}\text { Subject } \\
\text { ID }\end{array}$} & \multicolumn{2}{|c|}{1 Month FU } & \multicolumn{2}{|c|}{3 Month FU } \\
\hline & Usability & Outcome & Usability & Outcome \\
\hline 1 & 3 & 2 & 3 & 2 \\
\hline 2 & 3 & 3 & 3 & 3 \\
\hline 3 & 3 & 3 & 3 & 3 \\
\hline 4 & 3 & 2 & 3 & 3 \\
\hline 5 & 3 & 2 & 3 & 3 \\
\hline 6 & 3 & 1 & 3 & 2 \\
\hline 7 & 3 & 3 & 3 & 3 \\
\hline 8 & 3 & 3 & 3 & 3 \\
\hline 9 & 3 & 3 & 3 & 3 \\
\hline 10 & 3 & 2 & 3 & 3 \\
\hline 11 & 3 & 2 & 3 & 3 \\
\hline 12 & 3 & 3 & 3 & 3 \\
\hline 13 & 3 & 3 & 3 & 3 \\
\hline 14 & 3 & 2 & 3 & 3 \\
\hline 15 & 3 & 3 & 3 & 3 \\
\hline Mean & 3.0 & 2.5 & 3.0 & 2.9 \\
\hline
\end{tabular}

Despite the lower efficacy achieved by the Infinity home-use device on dark skin, when compared to light skin, subject satisfaction was very high. All subjects found the device ergonomic and easy to use. Most of the subjects were very satisfied with the treatments outcome in hair reduction.

In another study [9], a similar home-use device was used for only 3 sessions bi-monthly. As a result, lower percent hair reduction (40.4\%) was noted in the axillae 1 month after last treatment and it was reduced to $37.8 \%$ at 6 months follow-up. The skin types treated on axillae were II-III. According to this study, it may be worthwhile to check the percent hair reduction of the current study after 6 months.

No adverse events were recorded, like all IPL hair removal home-use devices that were reported to be safe. Some of the devices, however, had impaired efficacy due to safety [10]. In the current study efficacy was not affected, as the low optical energy was compensated by the galvanic energy used in the Infinity device. The galvanic energy which is applied in a continuous wave enables better absorption of the IPL pulses in the hair shaft by widening the skin pores [11]. As a result, less optical energy is needed to destroy the hair follicle, without creating damage to the surrounding dark skin.

\section{Conclusion}

Low-fluence pulsed light combined with galvanic energy, using the novel technology of eHPL ${ }^{\mathrm{TM}}$ may be applied safely and effectively for at home hair removal for subjects with dark skin type of V-VI. All patients (100\%) showed a reduction in hair removal at 1 and 3 months, with zero adverse effects.

\section{References}

[1] Gold, M.H., Bell, M.W., Foster, T.D. and Street, S. (1999) One Year Follow-Up Using an Intense Pulsed Light Source for Long Term Hair Removal. Journal of Cutaneous Laser Therapy, 1, 167-171. http://dx.doi.org/10.1080/14628839950516823

[2] Dierickx, C.C. (2002) Hair Removal by Lasers and Intense Pulsed Light Sources. Dermatologic Clinics, 20, $135-146$. http://dx.doi.org/10.1016/S0733-8635(03)00052-4 
[3] Gold, M.H. (2007) Lasers and Light Sources for the Removal of Unwanted Hair. Clinical Dermatology, 25, $443-453$. http://dx.doi.org/10.1016/j.clindermatol.2007.05.017

[4] Anderson, R.R. and Parish, J.A. (1983) Selective Photothermolysis: Precise Microsurgery by Selective Absorption of Pulse Radiation. Science, 20, 524. http://dx.doi.org/10.1126/science.6836297

[5] Grossman, M.C., Dierickx, C.C., Farineli, W., Flotte, T. and Anderson, R.R. (1996) Damage to Hair Follicles by Normal Mode Ruby Laser Pulses. Journal of American Academy of Dermatology, 35, 889-894. http://dx.doi.org/10.1016/S0190-9622(96)90111-5

[6] Johnson, F. and Dovale, M. (1999) Intense Pulsed Light Treatment of Hirsutism: Case Reports of Skin Phototypes V and VI. Journal of Cuteneous laser Therapy, 1, 233-237

[7] Gold, M.H., Foster, A. and Biron, J.A. (2010) Low-Energy Intense Pulsed Light for Hair Removal at Home. The Journal of Clinical and Aesthetic Dermatology, 3, 48-53.

[8] Gold, M.H., Biron, A.B. and Thompson, B. (2015) Clinical Evaluation of a Novel Intense Pulsed Light Source for Facial Skin Hair Removal for Home Use. The Journal of Clinical and Aesthetic Dermatology, 8, 30-35.

[9] Alster, T.S. and Tanzi, E.L. (2009) Effect of a Novel Low-Energy Pulsed-Light Device for Home-Use Hair Removal. Dermatologic Surgery, 35, 483-489. http://dx.doi.org/10.1111/j.1524-4725.2009.01089.x

[10] Mulholland, R.S. (2009) Silk'n' ${ }^{\mathrm{TM}}$ —A Novel Device Using Home Pulsed Light ${ }^{\mathrm{TM}}$ for Hair Removal at Home. Journal of Cosmetic and laser Therapy, 11, 106-109. http://dx.doi.org/10.1080/14764170902902806

[11] Town, G. and Ash, C. (2010) Are Home-Use Intense Pulsed Light (IPL) Devices Safe? Lasers in Medical Science, 25, 773-780. http://dx.doi.org/10.1007/s10103-010-0809-6

[12] Nordmann, L. (2010) Professional Beauty Therapy-The Official Guide to Level 3. 4th Edition, Cengage Learning EMEA, 231-237.

[13] Sadick, N.S. and Shaoul, J. (2004) Hair Removal Using a Combination of Conducted Radiofrequency and Optical Energies-An 18-Month Follow-Up. Journal of Cosmetics and Laser Therapy, 6, 21-26. http://dx.doi.org/10.1080/14764170410029013 